\title{
A critical analysis of alcohol hangover research methodology for surveys or studies of effects on cognition
}

\author{
Richard Stephens • James A. Grange • Kate Jones • \\ Lauren Owen
}

Received: 21 November 2013 / Accepted: 4 March 2014 /Published online: 16 March 2014

(C) Springer-Verlag Berlin Heidelberg 2014

\begin{abstract}
Rationale Alcohol hangover may be defined as an adverse effect of heavy alcohol consumption present after sufficient time has elapsed for the alcohol to have been eliminated from the blood. Understanding how hangover may impair performance is important for public safety; yet, there is relatively little hangover research. This paper outlines good practice for future studies.

Objectives This paper presents a critical analysis of hangover methodology for surveys or studies of effects on cognition with human subjects and provides suggestions for optimum research practice for laboratory-based and naturalistic alcohol hangover studies.

Results Four hangover symptom scales have been developed and subjected to psychometric testing. For retrospective assessment, we recommend the Hangover Symptoms Scale (HSS) or the Alcohol Hangover Severity Scale (AHSS). For concurrent assessment of hangover symptoms, we recommend either the Acute Hangover Scale (AHS), the five-item version of the HSS, or the AHSS. In research aiming to assess the cognitive effects of alcohol hangover, we suggest focusing on the cognitive domains of attention, memory and executive function, and we specify a number of tests within these cognitive domains that are likely to be sensitive to any decrements due to hangover. Finally, we argue that naturalistic studies should assess biological markers to improve the accuracy of estimates of alcohol consumption. Specifically, we recommend the assessment of ethyl glucuronide (EtG) for this purpose.
\end{abstract}

R. Stephens $(\bowtie) \cdot$ J. A. Grange $\cdot$ L. Owen

Centre for Psychological Research,

Keele University, Keele Staffordshire ST5 5BG, UK

e-mail: r.stephens@keele.ac.uk

K. Jones

Health and Safety Laboratory, Derbyshire, UK
Conclusions Recommendations are made with respect to assessing hangover symptoms, cognitive effects of hangover and biological markers of alcohol consumption.

Keywords Review $\cdot$ Alcohol hangover $\cdot$ Symptoms · Cognitive performance $\cdot$ Biological markers $\cdot$ Ethyl glucuronide

\section{Introduction}

Alcohol hangover may be defined as any adverse effect experienced following alcohol consumption after sufficient time has elapsed for the alcohol to have been eliminated from the blood (Verster et al. 2010). Hangover occurs due to some of the several biological mechanisms, which are not fully understood, including reduced blood glucose concentration, imbalance in the immune system and the metabolism of congeners (alcohols in drinks other than ethanol) such as methanol (Penning et al. 2010). Increased levels of acetaldehyde, dehydration, sleep deprivation and insufficient eating may also play a part (Verster et al. 2003).

Compared with research on acute and chronic effects of alcohol, alcohol hangover has been a relatively neglected research agenda until comparatively recently. This is now changing and several active alcohol hangover research teams in Europe and the USA came together in 2009 to form the Alcohol Hangover Research Group. The first meeting of the group resulted in a consensus statement on the best practice in alcohol hangover research (Verster et al. 2010). The outcome of this statement was to call for further research aimed at better understanding the alcohol hangover mechanisms, assessing economic effects of hangover, assessing individual differences in hangover susceptibility (including, age, sex and genetic factors), hangover remedies and developing research tools and methodologies. 
This paper presents a critical analysis of hangover methodology for research with human subjects and provides suggestions for optimum research practice for experimental alcohol hangover research. The specific focus of the paper is comparing the two main experimental methodologies applied to assess hangove - laboratory and naturalistic studies; assessing hangover symptoms; assessing cognitive effects of hangover; and assessing biological markers of alcohol consumption. This analysis will form a source of reference and guidance for alcohol hangover researchers and alcohol policy setters.

\section{Laboratory and naturalistic studies}

Literature reviews (Stephens et al. 2008; Ling et al. 2010) have identified 30 English language peer-reviewed laboratory studies that have investigated some aspects of psychological performance during alcohol hangover following controlled alcohol ingestion and four naturalistic studies that have assessed performance effects in participants tested the morning after an evening spent drinking and socialising.

A typical laboratory hangover study involves giving alcohol to one group of participants and placebo to a second group, although sometimes a within-subjects design can be applied so that the same participants undergo both treatments on separate days. Usually, a set dose of alcohol is administered relative to the body weight of the participant. The typical dose of $1 \mathrm{~g} / \mathrm{kg}$ is roughly equivalent to five $350 \mathrm{ml}$ bottles of $5 \%$ alcohol by volume (ABV) beer, assuming an average body weight of $70 \mathrm{~kg}$. This is usually administered as a vodka and orange beverage. The placebo drink is usually orange juice with a few drops of vodka floated on the surface. A time period is allowed for acute alcohol intoxication effects to dissipate (usually around $11 \mathrm{~h}$ ) during which participants may be retained in the laboratory for monitoring, or they may be allowed home to sleep. Then, participants are recalled for assessment using cognitive or other psychological performance tests. Electronic breath analysis is used to estimate blood alcohol level (BAL), and specifically to verify that the estimated BAL is zero or very close to zero, such that acute alcohol intoxication effects are no longer present. Any performance decrements observed the morning after alcohol consumption, compared with placebo, are taken to be alcohol hangover effects.

A typical naturalistic hangover study involves inviting participants to attend for assessment on a morning following an evening spent drinking and socialising, and, as a control condition, after an evening of abstinence. Naturalistic studies allow participants to choose the beverage, the quantity consumed, the setting, and whether to eat at the same time as drinking. All of these factors have been theorised as likely to influence alcohol and alcohol effects (Finnigan and Hammersley, 1992). While the mean amount of alcohol consumed in naturalistic studies is similar to the mean amount consumed in laboratory research (around $1 \mathrm{~g} / \mathrm{kg}$; Stephens et al. 2008), there is much greater variation in consumption with some participants choosing to drink a far higher amount than would be ethically permissible in the laboratory. As with laboratory studies, electronic breath analysis is used to verify that acute alcohol intoxication effects are no longer present at testing, and performance decrements observed the morning after alcohol consumption compared with after abstaining are taken to be alcohol hangover effects.

In our 2010 review (Ling et al. 2010), we suggested that both laboratory studies and naturalistic studies have an important role to play in furthering a scientific understanding of the alcohol hangover. We noted that recent laboratory studies have employed an improved procedure for dosing alcohol to more reliably produce hangover effects (Rohsenow et al. 2006; Howland et al. 2010; Rohsenow et al. 2010). It involves dosing to achieve a specified BAL rather than at a set dose per body weight. In practice, this consisted of first administering a guideline dose in grams per kilogram, and subsequently providing or withholding further alcohol depending upon electronic breath analysis readings, so that the desired BAL of $0.10-0.12 \mathrm{~g} \%$ breath alcohol is achieved (approximately equivalent to six $350 \mathrm{ml}$ bottles of $5 \% \mathrm{ABV}$ beer, assuming a body weight of $70 \mathrm{~kg}$ ). It is recommended that future laboratory studies of alcohol hangover should employ this more efficient procedure for administering controlled dosages of alcohol.

In our 2010 review, we also noted that the methodology for naturalistic hangover studies could be improved by reducing the extent to which expectancy effects contribute to symptom reporting and performance decrements in hangover conditions. We suggested that patterns of drinking across a regular week are predictable. For example, in some English universities, the two most popular nights for large-scale social events are Wednesdays and Fridays. Therefore, organising data collection on Thursday and Saturday mornings is possible to assess hangover effects in single-blind conditions; that is, without disclosing to the participants that the study is in any way concerned with alcohol hangover. Pilot work in our laboratory shows that a significant percentage of participants recruited in this way arrive at the laboratory with a hangover, but as they are blind to the study aim of assessing hangover effects, they are as close as possible to how hungover people behave in the real world, outside of the laboratory, aiming to have a reasonable go under the circumstances.

At the time of writing and since our most recent review was published (Ling et al., 2010), there appears to have been just one new study on the effects of hangover on human cognitive function (McKinney et al. 2012). This was ascertained from searching in October 2013 with the term 'hangover' in title in the databases, PsycINFO, EBSCO Psychology and Sociology Databases, Web of Knowledge and ScienceDirect, and by viewing articles that have cited some of the most influential 
reviews of alcohol hangover studies (Stephens et al. 2008; Prat et al. 2008; Howland et al. 2008; Swift and Davidson, 1998; Wiese et al. 2000; Verster, 2008).

McKinney et al. (2012) employed a naturalistic design in which 48 student participants ( 33 females) were tested on two occasions 1 week apart: once after an evening spent drinking a mean of 11.84 units (equivalent to a mean dose of $1.31 \mathrm{~g} / \mathrm{kg}$ assuming the typical person weighs $70 \mathrm{~kg}$ ) and once after an evening of abstention. The elapsed time between drinking and testing was $8-11 \mathrm{~h}$, and zero estimated blood alcohol concentration was verified at testing for all participants. Hangover decrements were observed for sustained attention (higher proportion of missed targets in the hangover condition) and selective attention (slower reaction time for more peripherally located flanker items in hangover state). There were no effects on divided attention or spatial attention. There was an observed slowed response on incongruent Stroop items in the hangover state.

\section{Assessing hangover symptoms}

Four hangover symptom scales have been developed and psychometrically tested: the Hangover Symptoms Scale, or HSS (Slutske et al. 2003; Robertson et al. 2012); a five-item version of the HSS known as the HSS-5 (Piasecki et al. 2010); the Acute Hangover Scale, or AHS (Rohsenow et al. 2007); and the Alcohol Hangover Severity Scale, or AHSS (Penning et al. 2013). Slutske's HSS was designed with the aim of assessing hangover symptom occurrence retrospectively. It contains 13 items: thirst, tiredness, headache, difficulty concentrating, nauseousness, weakness, sensitivity to light and sound, sweating, trouble sleeping, vomiting, anxiousness, trembling and depression. Respondents are asked to rate the percentage of times over the past year that a drinking occasion was followed the next morning by each symptom. Response is captured via five categories labeled $0-4$ with the anchors: ' $0 \%$ of the time' for ' 0 ' and ' $100 \%$ of the time' for ' 4 '. A dichotomous method of scoring such that a score of 1 is attained on any item with response higher than 0 (possible range of scores 0-13) was preferred because it yielded a more normal distribution. Cronbach's alpha for this method was 0.84 , indicating that the scale is reliable. Initial validation work was carried out with a sample of 1,230 college students. The argument for construct validity was based on correlational analyses showing correlations between HSS scores and frequency of drinking $(r=0.44)$, frequency of getting drunk ( $r=$ $0.52)$, typical quantity of alcohol consumed when drinking $(r$ $=0.40$ ), differences in symptom reporting between those with and without a personal history of alcohol-related problems $(d$ $=0.86)$ and family history of alcohol-related problems $(d=$ 0.29) (Slutske et al. 2003).

A more recent validation study of the HSS comprised a comparison of HSS scores for the previous 12 months with concurrent hangover ratings recorded by 404 participants over a 3-week period using electronic diaries. It was found that HSS scores relating to the previous 12 months predicted concurrent hangover reports, and this was demonstrated statistically by calculating an odds ratio, $\mathrm{OR}=2.11(95 \% \mathrm{CI}=$ 1.78-2.49). This statistic indicates that individuals reporting having had at least one of the HSS symptoms over the previous 12 months were twice as likely to report experiencing a hangover over the diary period than individuals who did not report having had at least one of the HSS symptoms over the previous 12 months (Robertson et al. 2012). In addition, a five-item version of the HSS (the HSS-5) has recently been developed for the concurrent assessment of hangover occurrence and severity in survey or diary studies (Piasecki et al. 2010). It includes the items 'more tired than usual', 'headache', 'nauseous', 'very weak' and 'extremely thirsty or dehydrated'. For each item, participants were asked to rate how they have felt in the past 15 min using a scale from 1 'not at all' to 5 'extremely'. Scores for each of the five items were raised on mornings following evening drinking where participants rated that they were experiencing a hangover-like experience. The amount of alcohol consumed was, on average, 10.1 drinks, approximately equivalent to a dose of $2.0 \mathrm{~g} / \mathrm{kg}$ assuming an average weight of $70 \mathrm{~kg}$. The scale developers did not, however, endorse an overall score.

Rohsenow's AHS was designed to assess concurrent hangover symptoms. The AHS asks respondents to rate each of the nine different hangover symptoms on a scale of $0-7$ with four anchors: ' 0 '='none', ' 1 '='mild', ' 4 ' ='moderate', and ' 7 ' ='incapacitating'. The symptoms are hangover, thirsty, tired, headache, dizziness, nausea, stomachache, heart racing and loss of appetite. The final score is obtained by adding up the scores for all nine items, and the range of possible scores is 0 63. The AHS was tested on a sample of 189 adults who drank $1-1.2 \mathrm{~g} / \mathrm{kg}$ of beer, vodka and cola or bourbon and cola on one occasion, and a placebo of non-alcoholic beer, or a mixture of tonic water and cola with a few drops of vodka or bourbon floated on top. The AHS was demonstrated to be reliable, with good split-half reliability, Cronbach's $\alpha=0.84$, and validity, such that the mean scale score differed largely in a withinsubjects comparison of participants who had and had not consumed alcohol the previous evening $(d=1.29)$. In addition, all of the AHS items were rated higher the morning after alcohol consumption compared with placebo, illustrating that the AHS is a sensitive instrument for the assessment of hangover symptoms.

The AHSS was designed to assess hangover severity rather than hangover incidence. Its items were derived from a review of all symptoms of hangover reported in the literature. An initial list of 47 items was reduced to 12 items in the final version via factor analysis of data from 791 survey respondents followed by regressing a short list of 21 items against overall hangover rating. The 12 items are fatigue, clumsiness, 
dizziness, apathy, sweating, shivering, confusion, stomach pain, nausea, concentration problems, heart pounding and thirst. These are assessed via a 10-point Likert scale. A subsequent survey of 966 participants verified that the AHSS is reliable (Cronbach's $\alpha=0.85$ ) and valid (correlation between AHSS and AHS was $r=0.92$ ). The AHSS was further developed by assessing reportage of hangover symptoms in a sample of 119 volunteers the morning after having consumed a mean of 9.7 alcoholic beverages producing an estimated peak blood alcohol concentration of $1.64 \%$, which is approximately $1.56 \mathrm{~g} / \mathrm{kg}$ assuming a $70 \mathrm{~kg}$ body weight. In a withinsubjects comparison with a morning following an evening of abstinence, the mean AHSS score was significantly raised after alcohol $(p<0.001)$, and all items differed significantly across the alcohol and no alcohol conditions. Interestingly, AHSS and AHS scores were highly correlated, $r=0.95$.

To summarise, there are four hangover symptom scales all of which have been shown to have good levels of reliability and validity. In choosing a scale for a particular application, a first question to ask is whether hangover symptoms are required to be assessed retrospectively or concurrently. The HSS (Slutske et al. 2003; Robertson et al. 2012) was developed for the assessment of hangover symptoms retrospectively over the time period of the previous 12 months. The AHS (Rohsenow et al. 2007) was developed to assess concurrent hangover symptoms. The AHSS (Penning et al. 2013) was developed to assess the severity of the most recent hangover event including a concurrent hangover. The HSS-5 (Piasecki et al. 2010) was developed to assess symptom severity for a concurrent hangover. When choosing a scale, a second question to ask is whether symptom occurrence and/or severity need to be assessed. The HSS is best deployed to assess the occurrence of symptoms, but its scoring method is less suited where it is important to measure hangover severity. On the other hand, the AHS, the HSS-5 and the AHSS were developed with scoring systems that reflect hangover severity as well as incidence. Indeed, for the concurrent assessment of hangover incidence and severity based on symptoms, there is little to choose between these three scales. All have demonstrated reliability and validity, and the AHS and AHSS are highly correlated. The HSS-5 is the briefest at five items, but this scale does not provide an overall hangover score. The AHS is slightly briefer at nine items, although at 12 items, the AHSS would not take significantly longer to administer. The AHS has been in existence for longer, and so, currently, there are more prior studies against which compare and contrast new data sets for the AHS. We recommend investigators to exercise their own judgment in choosing between the HSS-5, the AHS and the AHSS.

Assessing cognitive effects of hangover

The consensus statement on the best practice in alcohol hangover research (Verster et al. 2010) makes some general recommendations around methodology for assessing performance effects of alcohol hangover. These include the importance of selecting tests that are supported by psychometric data, the suggestion that tests that are relevant to everyday activities should be selected and the need to select tests that either have previously been shown to be sensitive to hangover (rather than acute alcohol effects) or have a mechanistic connection with hangover (Verster et al. 2010). We would concur with these suggestions, but here, we make some specific recommendations as to what tests are the most appropriate for the assessment of hangover effects on cognitive performance based on reviews of previous hangover studies.

In our reviews of the cognitive effects of alcohol hangover, we considered cognition from the perspectives of memory, attention, processing speed, executive function and psychomotor function (Stephens et al. 2008; Ling et al. 2010). In our most recent literature review, we reported that several laboratory and naturalistic studies had found specific cognitive decrements in attention (ability to pay attention and react to stimuli) and memory (ability to store and retrieve information) during the hangover phase of alcohol consumption. On the other hand, processing speed and psychomotor function have not been shown to be affected by hangover in laboratory or naturalistic studies.

We called for further research assessing attention and memory deficits of hangover in greater detail (Ling et al. 2010). Also, as of the review of Ling et al (2010), few studies had investigated hangover effects on executive functioning (the theorised control system in psychology that controls and manages other cognitive processes; e.g. Miyake et al. 2000). Ling et al. called for such research to be carried out for two reasons. One is that executive function decrements have been observed following experimentally induced sleep loss (Jones and Harrison, 2001), one of the mechanisms thought to underlie hangover. The other is that acute alcohol intoxication affects controlled processes more than automatic processes, implicating an effect on executive function (Fisk and Scerbo, 1987), and such an effect may persist into the hangover phase. A further reason to study executive function is its theoretical connection to 'higher level' cognitive processes, such as planning, overcoming habitual responding and error correction, among others (e.g. Miyake et al. 2000). Such functions are imperative for successful performance in many everyday tasks, and as such, it is important to assess the effects of alcohol hangover on these. The next sections focus on the assessment of attention, memory and executive function in alcohol hangover research.

\section{Attention}

There are a variety of theorised attention sub-systems based on functional and anatomical data. For example, Posner and Petersen (1990) theorised that attention sub-systems exist for signal detection, orienting to a sensory event and maintaining 
a vigilant state. In their critical review of attention deficits in Alzheimer's disease, Perry and Hodges (1999) elaborate on these and other theoretical accounts of attention to suggest three major aspects of attention that are useful to consider in the context of assessing attention function under challenging conditions. These are selective attention, sustained attention and divided attention. These same sub-divisions of the attention system were acknowledged in a more recent review of attention deficits arising from a variety of neurological conditions (Coulthard et al. 2006).

Selective attention is the ability to attend to a specified stimulus and to avoid distraction by other stimuli. Some classic measures of selective attention are the Stroop task, where participants are asked to name the ink colour of a series of colour words (e.g. the word 'blue' printed in red ink); cancellation tasks, in which participants are asked to delete a specified letter or digit in a list of letters or digits; search tasks, in which participants must locate specified target shapes out of an array of distracter shapes; 'flanker tasks', wherein participants must respond accordingly to a central target flanked by potentially task-irrelevant stimuli (e.g. the Eriksen Flanker Task; Eriksen and Eriksen, 1974); and the hidden figures task, in which participants must name large characters that are made up of smaller non-matching characters (e.g. a large number 1 made up of small number 4's) (Perry and Hodges, 1999).

Sustained attention is the ability to maintain attention on a task over a specified continuous time period. Tasks requiring the identification of infrequently appearing targets (also called vigilance tasks) measure the ability to sustain attention (Perry and Hodges, 1999). Examples of such tests are the Continuous Performance Task (e.g. Rosvold et al. 1956; Sjøgren et al. 2000), requiring the identification of specified letters, numbers, symbols or sounds appearing infrequently and randomly within a series of distracter letters, and 'Clock Jump' tasks (e.g. the Mackworth Clock Task) in which the second hand of a clock must be monitored for occasions when it moves forward by $2 \mathrm{~s}$ instead of by $1 \mathrm{~s}$ (Perry and Hodges, 1999). It is worth noting here that many cognitive tasks measure multiple aspects of cognition; for example, the Continuous Performance Task has been used to assess impulsivity as well as attention (Turgay et al. 2003).

Divided attention is the ability to attend to more than one stimulus simultaneously. The tasks involved may be from the differing or the same modality. Two tasks that make use of different modalities will avoid problems associated with the limited amount of attention that can be applied in any one modality and provide an assessment of higher level divided attention associated with executive functioning. An example of such a test of divided attention is Baddeley's combination of having participants track a moving object on screen (spatial modality) while at the same time reciting a span of digits (verbal/audio modality). On the other hand, the dichotic listening task requires participants to attend to two stimuli played individually into each ear through headphones (Perry and Hodges, 1999).

Assessment of attention in previous hangover research

Several well-controlled laboratory studies have detected attention decrements during alcohol hangover (see Table 1). Roehrs et al. (1991) showed divided attention decrements in a task that required participants simultaneously to track a moving target on a computer screen using a joystick and press a key in response to the appearance of a further target stimulus that could appear anywhere on the screen. The participants made a greater number of tracking errors $8 \mathrm{~h}$ after consuming a vodka, lime and tonic water drink containing $0.8 \mathrm{~g} / \mathrm{kg}$ of alcohol. Rohsenow et al. (2010) showed decrements (slowed reaction times) on two tasks of sustained attention. In the Continuous Performance Task, a series of letters appear singly on screen over a 5-min period at the rate of one per second, and participants must respond as quickly as possible to the letter ' $S$ ' (Baker et al. 1985). In the Psychomotor Vigilance Task, a series of digits appear on screen over a 5-min period at the rate of one per 3-7 s, and participants must respond as quickly as possible to all stimuli. Reaction time was slowed for both of these tests of sustained attention $10 \mathrm{~h}$ after drinking $1.1-1.2 \mathrm{~g} / \mathrm{kg}$ of neat bourbon or vodka and cola, compared with placebo. Howland et al. (2010) also observed slowed reaction time on the Psychomotor Vigilance Task $10 \mathrm{~h}$ after drinking $1.1 \mathrm{~g} / \mathrm{kg}$ of beer, compared with a zero alcohol beer control condition.

Attention decrements have additionally been shown in two naturalistic studies of hangover. Anderson and Dawson (1999) found decrements in letter and symbol cancellation tasks assessing sustained attention 12-16 h after consuming at least $1 \mathrm{~g} / \mathrm{kg}$ alcohol. However, as zero BAL at testing was not confirmed, these effects could be due to acute alcohol intoxication rather than hangover effects. In their recent naturalistic study, McKinney et al. (2012) used a related design and found impairments in tasks of sustained attention and selective attention in 48 participants tested the morning after consuming a mean of approximately $1.3 \mathrm{~g} / \mathrm{kg}$ of alcohol. BAL was zero at testing for all except two participants whose readings were very low. In the sustained attention task, participants were presented with a series of single digits on screen over $4 \mathrm{~min}$ and were asked to respond whenever three odd numbers appeared consecutively. When hung-over, participants more often failed to respond appropriately. The Eriksen Flanker Task (Eriksen and Eriksen, 1974) was one measure of selective attention. This required participants to respond to a letter ' $A$ ' or ' $B$ ' and to ignore other distracter letters that were similar or dissimilar to the target letters, and placed nearer or further away from the target letters. It was found that hungover participants were more susceptible to the confusion of having similar distracter letters, because when hungover, 


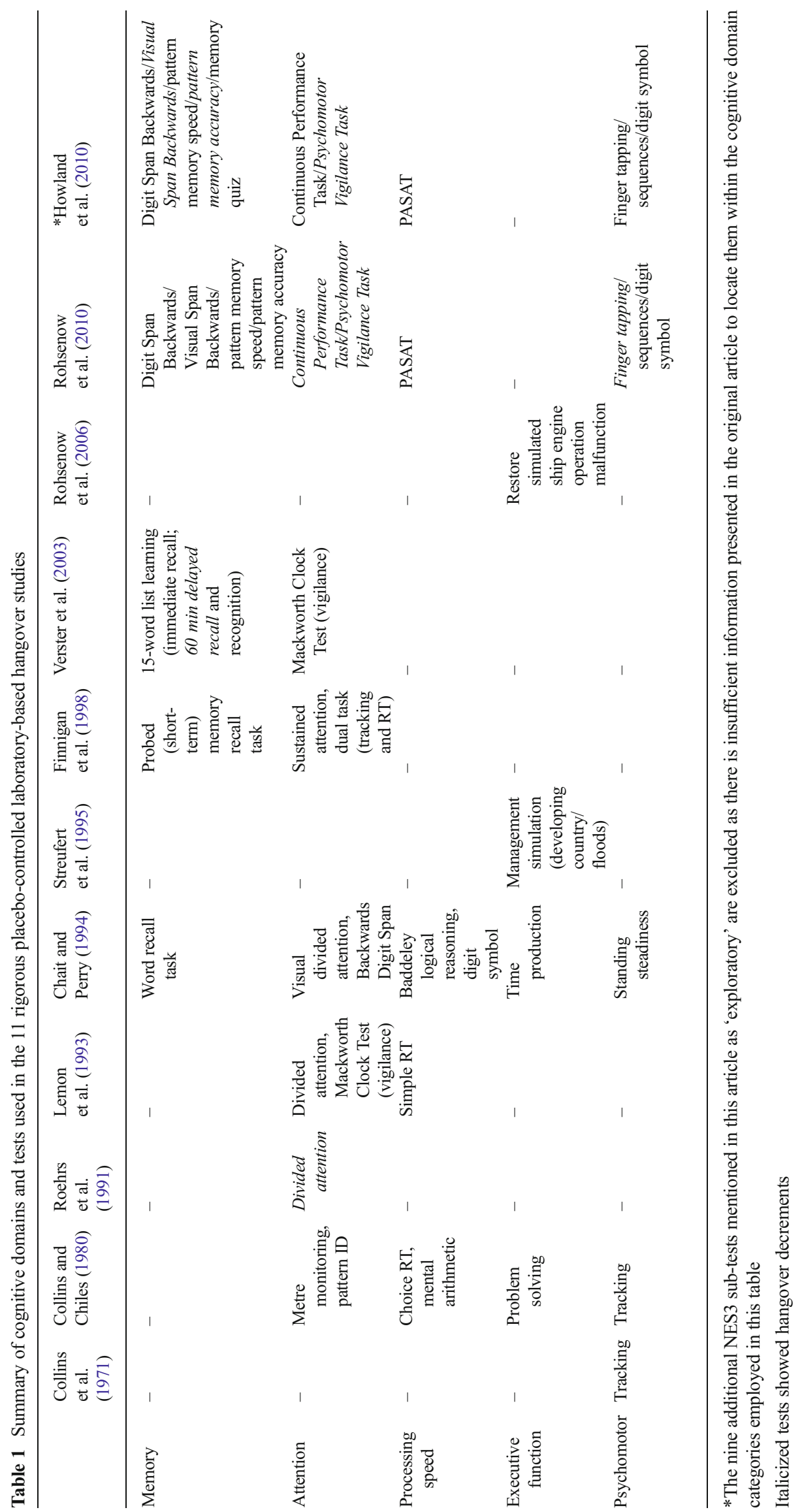


participants were slowed by incompatible distracters both nearer and further away from the targets, whereas when not hungover, only nearer compatible distracter letters slowed recognition of target letters. The Stroop task, which assesses selective attention among other aspects of cognitive function, required participants to state the ink colour of the words 'blue', 'red', 'green' and 'tan' written in either matching ink (e.g. 'red' written in red ink) or non-matching ink (e.g. 'red' written in blue ink). While the non-matching ink slows performance across the board, the degree of decrement was much more marked in the hangover condition.

Recommendations for assessing attention in future hangover research

Future hangover research aiming to detect cognitive effects of alcohol hangover should assess selective attention, sustained attention and divided attention. In the interests of replication, we would recommend any of the tests that have already been found to show hangover decrements, as described in the previous paragraphs. To summarise, for selective attention, versions of the Eriksen Flanker test or the Stroop test have been shown to be sensitive to hangover. For sustained attention, the Continuous Performance Task, the Psychomotor Vigilance Task, cancellation-type tasks and a task requiring response to three consecutive same stimuli have been shown to be sensitive to hangover. For divided attention, the Roehrs task requiring tracking a target on screen and responding to the onset of a second stimulus has been shown to be sensitive to hangover.

\section{Memory}

There is a wealth of research and theory pertaining to human memory. We are some way off a full understanding of memory, and therefore, one must be careful with terminology; for example, it is a matter of debate whether memory is best conceptualised as primarily an information storage system or an information-processing system encompassing storage (Rutherford et al. 2012). This section will summarise the most prominent theorised memory systems at the present time beginning with the theoretical distinction drawn between short-term and long-term memory. Short-term memory stores or processes are used to maintain information in the mind from moment to moment, e.g. remembering what you are supposed to be doing at a given time (Rutherford et al. 2012). Short-term memory lasts only as long as its content is being actively rehearsed, and is lost if attention shifts to some other stimulus. Baddeley's working memory model is the most prominent theory of short-term memory (Baddeley, 2012). The working memory model originally posited a three-component system in which two slave systems, the phonological loop and the visuospatial sketchpad, were coordinated by the central executive control mechanism. The two limited capacity slave systems are considered to facilitate storage and rehearsal of modality-specific information, with speech and acoustic details within the phonological loop and sight and geographical detail within the visuospatial sketchpad. The central executive control mechanism is thought to facilitate focusing of attention, dividing of attention, switching of task and interfacing with long-term memory. This latter function was via an additional sub-component, the episodic buffer that was incorporated into the model later. The attentional aspects of the central executive were considered earlier under 'attention'. The other aspects will be considered later under 'executive function'.

Long-term memory stores or processes are used to access information across timescales ranging from minutes to decades. Several long-term memory sub-systems have been proposed, and are reviewed by Rutherford et al. (2012). A key distinction is between declarative and non-declarative long-term memory (Squire, 1992). Non-declarative longterm memory refers to memories in the domains of skill learning, priming and associative learning including classical and operant conditioning and habituation. Within declarative long-term memory, a distinction is made between episodic and semantic memory. Episodic memories are those embedded in a specific time and place, usually experienced in relation to oneself. Semantic memories, on the other hand, are memories for facts or information without an autobiographical component. The most recent applications of this research are in the remember/know paradigm in which, following successful recall or recognition, the subject is asked to introspect as to the source of the memory, and specifically if they can remember being presented with a target (remember), or if they just have a strong feeling of familiarity for the target but cannot actually place themselves in relation to it (know).

It is currently under debate whether there is sufficient distinction between semantic and episodic memory to warrant their being treated as separate systems or different aspects of a single memory process. There are some good dissociative findings from research with amnesiacs, who tend more often to show deficits in episodic rather than semantic memory, although a distinction is less clear-cut in functional magnetic resonance imaging studies attempting to locate discrete neural substrates for the two memory types (Rutherford et al. 2012). Within declarative memory, a further distinction may be drawn between explicit and implicit memory (Schacter 1987). Explicit memory is recall or recognition for target items under conditions such that the subjects are aware that they are engaged upon a memory task. On the other hand, implicit memory refers to recognition or recall occurring outside of the conscious awareness of the subject, such that performance on a task may be observably affected by previous experience without the subject realising. However, it should be noted that a computational model which assumes no independence of 
implicit and explicit memory systems fits human data incredibly well, including data from neuropsychological dissociation studies which have been used to argue for different implicit/explicit memory systems (see Berry et al. 2008, for an excellent overview of this model).

A further distinction in long-term memory is that between retrospective memory, which pertains to events or information from the past, and prospective memory, which is the ability to perform a future action at a pre-determined time (Heffernan et al. 2010).

\section{Assessment of memory in previous hangover research}

In the hangover literature, long-term memory has been assessed using word list tasks that predominantly tap into episodic memory. Long-term memory has been assessed in three well-controlled laboratory studies and two naturalistic studies lacking a placebo control (see Tables 1 and 2), although only one of these studies showed effects. Verster et al. (2003) showed poorer 1-h delayed recall of items from a 15word list in 24 participants the morning after they had consumed $1.4 \mathrm{~g} / \mathrm{kg}$ of alcohol (mean 9.4 items recalled; SD 3.4) compared with placebo (mean 11.5 items recalled; SD 3.5). On the other hand, Chait and Perry (1994) did not show any decrement in a similar task $10 \mathrm{~h}$ following ingestion of $1.2 \mathrm{~g} /$ $\mathrm{kg}$ of alcohol under laboratory conditions, although this was most likely due to the low power afforded by a smaller sample size of 14 participants. Howland et al. (2010) did not show a decrement in a 30-question quiz, completed $9 \mathrm{~h}$ after the ingestion under laboratory conditions of $1.2 \mathrm{~g} / \mathrm{kg}$ of alcohol in a sample of 193 university students. The quiz was based on a video-taped lecture that had been observed the previous day while sober. In research employing naturalistic designs, immediate word recall and 1-h delayed word recognition were found to be reduced in two studies (McKinney and Coyle, 2004; 2007). Across the two studies, testing was at least $7 \mathrm{~h}$ after reported consumption of an average of $1.5 \mathrm{~g} / \mathrm{kg}$ of alcohol, and BAL was zero at testing for all except a few participants whose readings were very low. Immediate and delayed word recall and recognition tasks such as these are generally held to be tasks of episodic long-term memory; in so far as it has been assessed, the evidence points to hangover affecting this cognitive domain.

Short-term memory has generally not been found to be adversely affected across three well-controlled laboratory studies and one naturalistic study lacking a placebo control (see Tables 1 and 2). In the laboratory, Finnigan et al. (1998) used a probed test of short-term memory in which participants were asked to say which of a series of 10 consonants presented at 1-s intervals preceded a probe consonant from the list subsequently presented. Performance of 40 male volunteers was not adversely affected $10 \mathrm{~h}$ following the ingestion of approximately $0.7 \mathrm{~g} / \mathrm{kg}$ of ethanol. Rohsenow et al. (2010) did not show decrements in tests of digit- or visual pattern-based tests of short-term memory in the laboratory $10 \mathrm{~h}$ after the consumption of $1.2 \mathrm{~g} / \mathrm{kg}$ alcohol in a sample of 95 university students or recent graduates. Howland et al. (2010), however, did show short-term memory decrements on the same tests of digit- and visual pattern-based tests of short-term memory $9 \mathrm{~h}$ after the ingestion under laboratory conditions of $1.2 \mathrm{~g} / \mathrm{kg}$ of alcohol in a sample of 193 university students. The one naturalistic study of short-term memory and hangover did not show a decrement in 25 volunteers at an unspecified interval following the ingestion of an average of $1.75 \mathrm{~g} / \mathrm{kg}$ alcohol. The task was the same as that used by Finnigan et al. (1998). Therefore, across the board, there is little evidence of short-term memory decrements during alcohol hangover.

\section{Recommendations for assessing memory in future hangover research}

There is evidence for a deficit in episodic long-term memory during alcohol hangover according to the several studies that have employed word recall and recognition tasks. However, given the many other sub-types of long-term memory that have been theorised (see earlier section), there is a strong argument to broaden out the tests used to assess long-term memory effects of hangover to cover these other facets. A key research agenda would be an extension into tests of episodic memory employing the 'remember/know' paradigm in order to assess the relative contribution of deficits in recollection and/or familiarity to hangover decrements. Furthermore, no hangover studies have yet explored whether explicit and implicit long-term memory are affected by hangover, and no studies have assessed hangover effects on prospective memory, or aspects of non-declarative long-term memory such as skill learning, priming, associative learning or habituation.

Deficits in tests of short-term memory have been absent when these have been assessed in prior studies, but these studies utilised experimental paradigms that only required storage of information (so-called simple span tasks). Simple span tasks are not predictive of individual differences in higher level cognitive abilities (Süß et al. 2002), and as such might not be expected to be affected by hangover. Therefore, it is recommended that future alcohol hangover research assess the effects on working memory (c.f. short-term memory) using 'complex span' paradigms, which require online processing and manipulation of information, as well as storage. For example, in the operation span task, participants are presented with a series of individual letters that must be retained for future recall. However, after the presentation of each list item, participants must solve a mathematical problem; thus, the memoranda must be maintained whilst taskirrelevant processing is occurring (see Conway et al. 2005, for a thorough review on complex working memory span tasks). Using such complex span tasks in hangover research might 


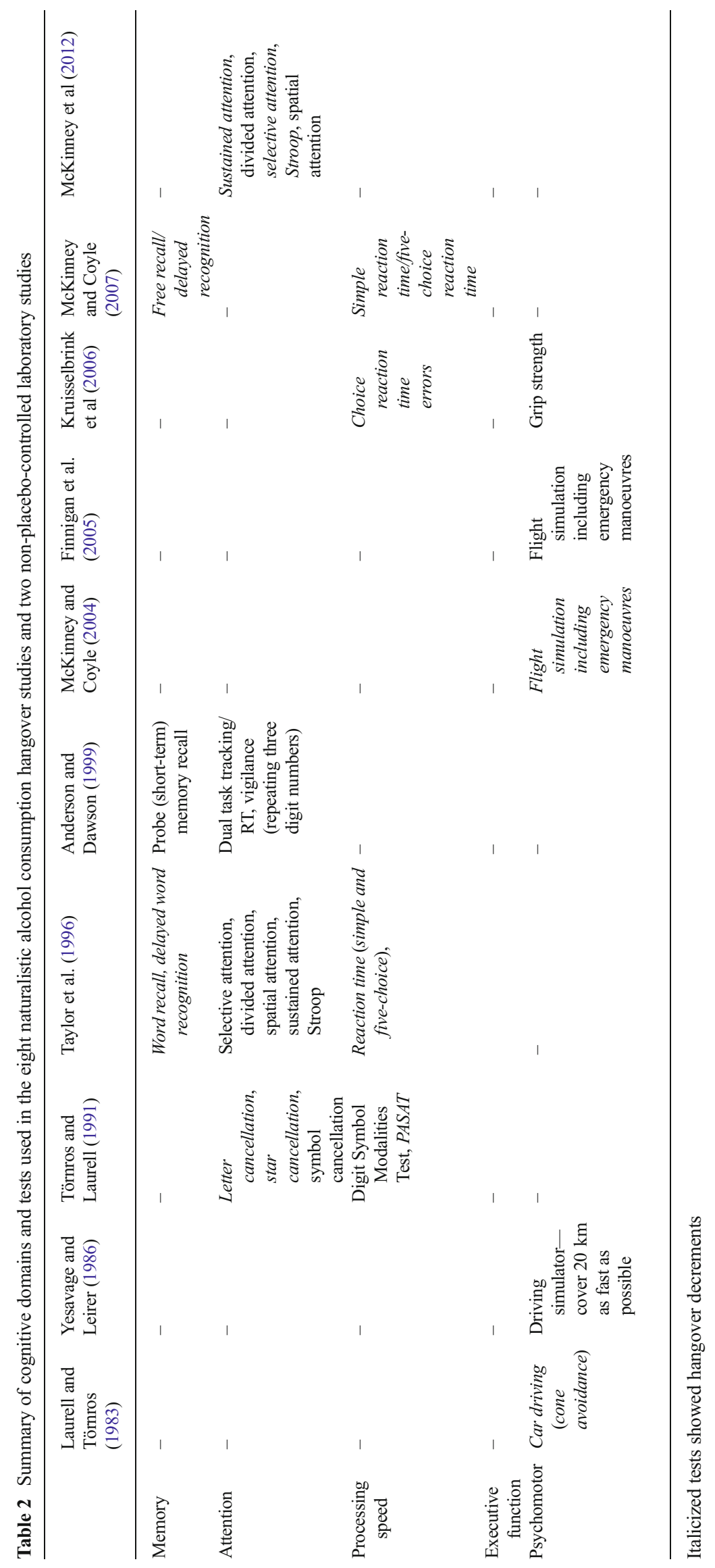


highlight effects of hangover on higher level cognitive processes (see also the section below).

\section{Executive function}

Executive functions (EFs) are a hypothesised set of control functions in the cognitive system-often associated with the frontal lobes (Norman and Shallice, 1986)-which directly regulate subordinate cognitive processes in order to achieve goal-directed behaviour. Successful performance on any complex task requires EFs, as the control and coordination of many individual cognitive processes are necessary. Driving, for example, presents us with an incredibly rich environment wherein many subordinate processes, such as perception (for obstacle detection/avoidance), memory retrieval (where am I going?), motor coordination etc., must be selected and controlled in a goal-directed manner in order to arrive safely at our destination.

Rather than being seen as a unitary process, EFs are generally thought to comprise a set of correlated, yet independent, functions. In their seminal individual differences study, Miyake et al. (2000) conducted confirmatory factor analysis on outcome measures from a battery of tasks thought to tap $\mathrm{EFs}$, and found a model with three independent factors best fitted the data collected. These three components-task switching, memory monitoring/updating and inhibition of pre-potent responses - were suggested by Miyake et al. to be the key roles of EF processes, with each contributing differentially to successful performance on more complex tasks.

Task switching is an important function in everyday life, allowing the cognitive system to act flexibly in dynamic environments (Grange and Houghton, in press); for example, if the telephone rings whilst engaged in writing a shopping list, the system must be able to switch from its current task (writing) to the now-relevant task (answering the phone). Failure to disengage from the irrelevant task leads to perseveration of action, a typical symptom of frontal lobe damage (Norman and Shallice, 1986). In the laboratory, several variants of the so-called 'task-switching paradigm' have been developed that aim to elucidate the cognitive processes that serve task-switching, and require participants switching back and forth between simple cognitive operations (such as parity and magnitude judgments on number stimuli; see Kiesel et al. 2010; Vandierendonck et al. 2010).

Memory monitoring and updating are also essential functions, and pertain to the need to monitor incoming information for task relevance, and update working memory representations accordingly. In contrast to short-term memory-which can be seen more as a passive store of information over the short-term - working memory is essential to store and manipulate information. This EF is essential for complex tasks; for example, mental arithmetic often involves holding intermediate values whilst working through a problem, and these values need to be updated (with the old values being removed) as the problem progresses. In the lab, a popular task measuring this ability is the $N$-back task (e.g. Jaeggi et al. 2010), in which participants must scan a stream of items and respond accordingly when the current item matches the item $N$ items ago (where increasing $N$ increases the load on memory).

Inhibition of pre-potent responses refers to the ability to suppress automatic responses to stimuli when that action is not task-relevant (or is just not required). Such inhibition is often essential in daily life; for example, when crossing the road, it is pertinent to stop your action of walking if you suddenly see a large vehicle speeding around the corner. Common empirical tasks to assess this function are the Stroop task (where the dominant response of word reading must be suppressed in favour of the weaker task of ink-colour naming, e.g. MacLeod, 1991) and the stop-signal task (where a response to a primary task must be suppressed before completion in response to a 'stop-signal'; e.g. Verbruggen and Logan 2008).

\section{Assessment of executive function in previous hangover research}

Four laboratory studies have assessed executive function during hangover. Collins and Chiles (1980) assessed performance of a test requiring participants simultaneously to complete several tasks. This involved complex workload performance requiring time-sharing skills. Three tasks were ongoing at any one time. Two of these were always the same: they were a choice reaction time task with five stimuli and a monitoring task with four stimuli that needed to be periodically corrected upwards or downwards. In addition, two of the three other tasks were also required to be carried out. The three additional tasks were mental arithmetic, pattern recognition and monitoring task with a single stimulus. The test lasted for $1 \mathrm{~h}$. Eleven aviation pilots aged $22-55$ years, including men and women, made up the participant group. There were three conditions: placebo, vodka and bourbon mixed with 7-Up. A within-subjects placebo control condition was applied. The dose of $3.25 \mathrm{ml} / \mathrm{kg}(2.57 \mathrm{~g} / \mathrm{kg})$ was one of the highest in any laboratory hangover studies, equivalent to 13 bottles of beer for an average person weighing $70 \mathrm{~kg}$. Electronic breath analysis readings showed that all participants had zero estimated blood alcohol when tested the next morning, after an interval of $7.5 \mathrm{~h}$. Despite the large dose and relatively short interval between consumption and testing, no adverse performance effects were observed during hangover.

Chait and Perry (1994) had 14 participants carry out a time production task $10 \mathrm{~h}$ following consumption of $1-1.2 \mathrm{~g} / \mathrm{kg}$ of alcohol administered as a neat ethanol, lime and tonic beverage, equivalent to $5-6$ bottles of beer for a $70-\mathrm{kg}$ person. A within-subjects placebo control condition was applied. The task required participants to estimate the passage of 30, 60 and $120 \mathrm{~s}$. Time production tasks have been linked with the 
sequencing and ordering aspects of executive function (Brown, 2006). There were no next morning differences in time estimation for the alcohol condition compared with placebo. Zero estimated blood alcohol at testing was verified.

Streufert et al. (1995) had 21 participants carry out a managerial effectiveness task $12 \mathrm{~h}$ after consuming $1 \mathrm{~g} / \mathrm{kg}$ alcohol as a neat ethanol, tonic and peppermint beverage, equivalent to five bottles of beer for an average person weighing $70 \mathrm{~kg}$. Zero estimated blood alcohol was verified at testing, and a within-subjects placebo control condition was applied. In the task, which lasted for $6 \mathrm{~h}$, participants had to take decisions around deploying resources in order to manage two scenarios: governing a developing country with economic problems and coordinating a disaster control centre in response to heavy rain and flooding. This task also requires the sequencing and ordering aspects of executive function. They were assessed on five variables: breadth of approach, basic planning/strategy, integrative planning/strategy, creativity of response and over-planning. There were no performance decrements in the hangover condition in comparison to the placebo.

In a between-subjects design, Rohsenow et al. (2006) had 61 maritime academy cadets aged 21-26 years operate a simulated ship power plant. Participants drank $1.2 \mathrm{~g} / \mathrm{kg}$ of beer, equivalent to six bottles for a person weighing $70 \mathrm{~kg}$, or a placebo consisting of alcohol-free beer. The hangover test session was the next morning, a minimum of $10.5 \mathrm{~h}$ following the alcohol administration. Zero (or very close to zero) estimated blood alcohol was verified at testing. During the simulation, either the boiler or the turbo generator malfunctioned, and the participant was required to identify the problem and carry out remedial action. They were timed on how rapidly they were able to do this. There was no difference in the time taken for the alcoholic and non-alcoholic beer groups.

\section{Recommendations for assessing executive function in future hangover research}

Research on EFs is complicated by the fact that the functions under investigation are themselves unobservable (i.e. latent); as such, their influence can only be inferred via changes in manifest variables. Relatedly, performance on EF tasks is never a pure measure of EFs, as the tasks used to tap these EFs also measure subordinate cognitive processes. In the Stroop task, for example, the EF inhibition of pre-potent responses is required for successful performance, but so too are stimulus perception (i.e. visuospatial processing), response programming, memory retrieval and response execution, any one of which could be affected by an experimental manipulation. In hangover research, therefore, poor performance from a 'hangover' condition on the Stroop task should not automatically be used to conclude that hangover affects EFs; hangover could have affected one (or many) of the subordinate cognitive processes that the executive controls (perhaps hangover really just affects visuospatial processing, for example). Miyake et al. (2000) refer to this as the "task impurity problem", and it is a perennial challenge for EF research to circumvent. Miyake et al. addressed this problem by testing participants on a range of cognitive tasks thought to separately tap each EF, and statistically examining performance at the level of latent variables (by conducting factor analysis), but there are other options.

Another approach — one that we strongly recommend - is to fit computational/mathematical cognitive models to empirical data (Farrell and Lewandowsky, 2010). Such process models of specific EFs typically have parameters that reflect the latent variables of interest, and researchers can therefore examine the effects (if any) of hangover on these psychologically interesting variables. There are many successful models of individual EFs that could be utilised in this endeavour, too many to list here. One such example, the horse-race model of stop-signal performance (Logan and Cowan, 1984; Verbruggen and Logan, 2009; see Verbruggen et al. 2013, for an important discussion on the validity of different methods of fitting this model) allows estimation of stopsignal response time (SSRT), which is the latency of an unobservable process which stops a primary action: faster SSRT leads to faster-and hence more successful—stopping of an unwanted action that has already been initiated. Fitting such process models to data from hangover studies will additionally allow the researcher to address deeper questions than just whether groups differ at the level of manifest variables.

\section{Biological indicators}

One further aspect of improving hangover research methodology for naturalistic studies would be the inclusion of alcohol biomarker measurements in order to estimate alcohol consumption in participants. Although, as mentioned earlier, there is no significant difference in the mean reported alcohol consumption across laboratory and naturalistic designs; a key feature of naturalistic studies is that variation in consumption increases, with markedly higher maximum estimates. However, in naturalistic studies such estimates are based on recall of the participants - it is reasonable to assume that in a naturalistic setting, especially if the purpose of the study has been blinded, the recall of alcohol consumption may not be accurate. Most hangover studies reported in the past 10 years have included the measurement of BAL to ensure that acute alcohol intoxication is not being experienced by participants prior to testing. However, the use of other alcohol biomarkers has not been reported in hangover studies. There are a number of potential biomarkers arising from the main pathways of ethanol metabolism — oxidative and non-oxidative direct biomarkers and products of tissue damage resulting from alcohol 
consumption. Some, such as carbohydrate deficient transferrin, haemoglobin-bound acetaldehyde, fatty acid ethyl esters and phosphatidylethanol, are able to determine heavy alcohol use. However, these are generally long-term markers (requiring heavy alcohol consumption for at least 2 weeks before showing an effect) and may only have a role in studies where long-term alcohol habits, or the effect of such habits on hangover propensity/symptoms, are being investigated.

Ethyl glucuronide (EtG) and ethyl sulphate (EtS) are sensitive and specific biomarkers of alcohol consumption; they are complementary markers and are often measured together. Both are short-term markers and are detectable for about 5 days after alcohol consumption. They are also measurable in a number of body fluids (and hair) meaning that longer term consumption can also be assessed. The value of a marker such as EtG is that its elimination reflects that of alcohol (peak excretion is about $3 \mathrm{~h}$ after that of alcohol) and that it shows limited inter-individual variation in metabolism due to factors such as age, gender and ethnicity, allowing a more reliable reconstruction of dose. There have been a number of controlled volunteer studies looking at the toxicokinetics of EtG excretion after ethanol consumption (Høiseth et al. 2008, 2010; Halter et al. 2008; Dahl et al. 2002) at a dose of $0.5 \mathrm{~g} /$ $\mathrm{kg}$. These studies are in reasonable agreement that the peak excretion is $\sim 5 \mathrm{~h}$ after ethanol ingestion, with a maximum urine concentration of between 38 and $147 \mathrm{mg} / \mathrm{l}$, based on 40 volunteers across four studies. Elimination half-life was $\sim 2.5 \mathrm{~h}$. It is noted that urinary EtG concentration is affected by diuresis (Goll et al. 2002; Dahl et al. 2002), and therefore, creatinine-corrected results should be used, but not all the volunteer studies report such results. Although there is quite some variation in the peak excretion of EtG reported after a fixed dose of $0.5 \mathrm{~g} / \mathrm{kg}$ in these studies, it appears that such inter-individual variation is greatly reduced when looking at samples provided 10 to $20 \mathrm{~h}$ after ingestion - this is the time window of interest for hangover studies. Variation is also reduced when using creatinine correction. This makes the possibility of 'back-calculating' ethanol doses a reasonable enterprise; indeed, a kinetic model has already been described (Droenner et al. 2002) to prospectively calculate serum EtG concentrations based on ethanol doses (including multiple doses) and drinking times. Verifying participants' recall of alcohol consumption would therefore be possible if EtG were routinely used in naturalistic studies when looking at effects within $24 \mathrm{~h}$ of the drinking episode.

\section{Recommendations for assessing biological indicators in future hangover research}

Although EtG is widely reported in the scientific literature with regard to alcohol research, this is mostly focused on workplace alcohol testing and abstinence programs. There are only two references to hangover research - one looking at potential intoxication of admissions to accident and emergency hospital departments. The other (Lewis et al. 2013) reported that ethyl glucuronide can cause TLR4-dependent pain and that this could have implications for human conditions such as hangover headache. This raises the future research potential of EtG as a marker of both consumption and effect and whether the level of EtG at the time of the hangover study is correlated to any effects observed within the study. Such a finding would apply to all study designs. Given all of this, we strongly recommend the assessment of EtG in future alcohol hangover research.

\section{Conclusion}

This review has defined the two main study types in alcohol hangover research-laboratory-based and naturalistic designs - and discussed recent developments in hangover symptom assessment, assessing cognitive effects of alcohol hangover and assessing biological indicators in naturalistic studies of alcohol hangover. For retrospective assessment, we recommend the Hangover Symptoms Scale (HSS) or the Alcohol Hangover Severity Scale (AHSS). For concurrent assessment of hangover symptoms, we recommend either the Acute Hangover Scale (AHS), the five-item version of the HSS (HSS-5) or the AHSS. For research aiming to assess the cognitive effects of alcohol hangover, we suggest focusing on the cognitive domains of attention, memory and executive function. We specify a number of tests within these cognitive domains that are likely to be sensitive to any decrements due to hangover. Finally, we argue that naturalistic studies should assess biological markers to improve the accuracy of estimates of alcohol consumption. Specifically, we recommend the assessment of ethyl glucuronide (EtG) for this purpose.

Acknowledgment This research was supported by EU 7th Framework Marie Curie Research Fellowship Project No: 301424.

\section{References}

Anderson S, Dawson J (1999) Neuropsychological correlates of alcoholic hangover. S Afr J Sci 95:145-147

Baddeley A (2012) Working memory: theories models and controversies. Annu Rev Psychol 63:1-29

Baker EL, Letz RE, Fidler AT, Stuart S, Plantamura D, Lyndon M (1985) A computer-based neurobehavioral evaluation system for occupational and environmental pidemiology: methodology and validation studies. Neurotoxicol Teratol 7:369-377

Berry CJ, Shanks DR, Henson RN (2008) A unitary signal-detection model of implicit and explicit memory. Trends Cogn Sci 12:367373

Brown SW (2006) Timing and executive function: bidirectional interference between concurrent temporal production and randomization tasks. Mem Cogn 34:1464-1471 
Chait LD, Perry JL (1994) Acute and residual effects of alcohol and marijuana alone and in combination on mood and performance. Psychopharmacology 115:340-349

Collins WE, Schroeder DJ, Gilson RD, Guedry FE (1971) Effects of alcohol ingestion on tracking performance during angular acceleration. J Appl Psychol 55:559-563

Collins WE, Chiles WD (1980) Laboratory performance during acute intoxication and hangover. Hum Factors 22:445-462

Conway ARA, Kane MJ, Bunting MF, Hambrick DZ, Wilhelm O, Engle RW (2005) Working memory span tasks: A methodological review and user's guide. Psychon Bull Rev 12:769-786

Coulthard E, Singh-Curry V, Husain M (2006) Treatment of attention deficits in neurological disorders. Curr Opin Neurol 19:613-618

Dahl H, Stephanson N, Beck O, Helander A (2002) Comparison of urinary excretion characteristics of ethanol and ethyl glucuronide. J Anal Toxicol 26:201-204

Droenner P, Schmitt G, Aderjan R, Zimmer HA (2002) Kinetic model describing the pharmacokinetics of ethyl glucuronide in humans. Forensic Sci Int 28:24-29

Eriksen BA, Eriksen CW (1974) Effects of noise letters upon identification of a target letter in a non-search task. Percept Psychophys 16: 143-149

Farrell S, Lewandowsky S (2010) Computational models as aids to better reasoning in psychology. Curr Dir Psychol Sci 19:329-335

Finnigan F, Hammersley R (1992) The effects of alcohol on performance. In: Smith AP, Jones DM (eds) Handbook of human performance. Academic, London, pp 73-126

Finnigan F, Hammersley R, Cooper T (1998) An examination of next-day hangover effects after a $100 \mathrm{mg} / 100 \mathrm{ml}$ dose of alcohol in heavy social drinkers. Addiction 93:1829-1838

Finnigan F, Schulze D, Smallwood J, Helander A (2005) The effects of self-administered alcohol-induced 'hangover' in a naturalistic setting on psychomotor and cognitive performance and subjective state. Addiction 100:1680-1689

Fisk AD, Scerbo MW (1987) Automatic and control processing approach to interpreting vigilance performance: a review and reevaluation. Hum Factors 29:653-660

Goll M, Schmitt G, Ganssmann B, Aderjan RE (2002) Excretion profiles of ethyl glucuronide in human urine after internal dilution. J Anal Toxicol 26:262-266

Grange JA, Houghton G (in press) Task switching and cognitive control New York NY: Oxford University Press.

Halter CC, Dresen S, Auwaerter V, Wurst FM, Weinmann W (2008) Kinetics in serum and urinary excretion of ethyl sulfate and ethyl glucuronide after medium dose ethanol intake. Int J Legal Med 122: $123-128$

Heffernan T, Clark R, Bartholomew J, Ling J, Stephens R (2010) Does binge drinking in teenagers affect their everyday prospective memory? Drug Alcohol Depend 109:73-78

Høiseth G, Bernard JP, Stephanson N, Normann PT, Christophersen AS, Mørland J, Helander A (2008) Comparison between the urinary alcohol markers EtG EtS and GTOL/5-HIAA in a controlled drinking experiment. Alcohol Alcohol 43:187-191

Høiseth G, Yttredal B, Karinen R, Gjerde H, Mørland J, Christophersen AS (2010) A Ethyl glucuronide concentrations in oral fluid blood and urine after volunteers drank 05 and $10 \mathrm{~g} / \mathrm{kg}$ doses of ethanol. J Anal Toxicol 34:319-324

Howland J, Rohsenow DJ, Edwards EM (2008) Are some drinkers resistant to hangover? A literature review. Curr Drug Abuse Rev $1: 42-46$

Howland J, Rohsenow DJ, Greece JA, Littlefield CA, Almeida A (2010) The effects of binge drinking on college students' next-day academic test-taking performance and mood state. Addiction 105:655-665

Jaeggi SM, Buschkuehl M, Perrig WJ, Meier B (2010) The concurrent validity of the $\mathrm{N}$-back task as a working memory measure. Memory $18: 394-412$
Jones K, Harrison Y (2001) Frontal lobe function, sleep loss and fragmented sleep. Sleep Med Rev 5:463-475

Kiesel A, Steinhauser M, Wendt M, Falkstein M, Jost K, Philipp A, Koch I (2010) Control and interference in task switching - a review. Psychol Bull 136:849-874

Kruisselbrink LD, Martin KL, Megeney M (2006) Physical and psychomotor functioning of females the morning after consuming low to moderate quantities of beer. J Stud Alcohol 67:416-420

Laurell H, Törnros J (1983) Investigation of alcoholic hangover effects on driving performance. Blutalkohol 20:489-499

Lemon J, Chester G, Fox A, Greeley J, Nabke C (1993) Investigation of the "hangover" effects of an acute dose of alcohol on psychomotor performance. Alcohol Clin Exp Res 17:665-668

Lewis SS, Hutchinson MR, Zhang Y, Hund DK, Maier SF, Rice KC, Watkins LR (2013) Glucuronic acid and the ethanol metabolite ethyl-glucuronide cause toll-like receptor 4 activation and enhanced pain. Brain Behav Immun 30:24-32

Ling J, Stephens R, Heffernan TM (2010) Cognitive and psychomotor performance during alcohol hangover. Curr Drug Abuse Rev 3:80-87

Logan GD, Cowan WB (1984) On the ability to inhibit thought and action: a theory of an act of control. Psychol Rev 91:295-327

MacLeod CM (1991) Half a century of research on the Stroop effect: an integrative review. Psychol Bull 109:163-203

McKinney A, Coyle K (2007) Next-day effects of alcohol and an additional stressor on memory and psychomotor performance. J Stud Alcohol 68:446-454

McKinney A, Coyle K (2004) Next day effects of a normal night's drinking on memory and psychomotor performance. Alcohol Alcohol 39:509-513

McKinney A, Coyle K, Penning R, Verster JC (2012) Next day effects of naturalistic alcohol consumption on tasks of attention. Human Psychopharmacol-Clin Exp 27:587-594

Miyake A, Friedman NP, Emerson MJ, Witzki AH, Howerter A, Wager TD (2000) The unity and diversity of executive functions and their contributions to complex "frontal lobe" tasks: a latent variable analysis. Cogn Psychol 41:49-100

Norman DA, Shallice T (1986) Attention to action: willed and automatic control of behavior. In: Davidson RJ, Schwartz GE, Shapiro D (eds) Consciousness and self-regulation: advances in research and theory vol 4. Plenum, New York

Penning R, van Nuland M, Fliervoet LAL, Olivier B, Verster JC (2010) The pathology of alcohol hangover. Curr Drug Abuse Rev 3:68-75

Penning R, McKinney A, Bus LS, Olivier B, Slot K, Verster JC (2013) Measurement of alcohol hangover severity: development of the Alcohol Hangover Severity Scale (AHSS). Psychopharmacology 225:803-810

Perry RJ, Hodges JR (1999) Attention and executive deficits in Alzheimer's disease. Crit Rev Brain 122:383-404

Piasecki TM, Slutske WS, Wood PK, Hunt-Carter EE (2010) Frequency and correlates of diary-measured hangoverlike experiences in a college sample. Psychol Addict Behav 24:163-169

Posner MI, Petersen SE (1990) The attention system of the human brain. Annu Rev Neurosci 13:25-42

Prat G, Adan A, Pérez-Pàmies M, Sànchez-Turet M (2008) Neurocognitive effects of alcohol hangover. Addict Behav 33:15-23

Robertson BM, Piasecki TM, Slutske WS, Wood PK, Sher KJ, Shiffman S, Heath AC (2012) Validity of the hangover symptoms scale: evidence from an electronic diary study. Alcohol Clin Exp Res 36: $171-177$

Roehrs T, Yoon J, Roth T (1991) Nocturnal and next-day effects of ethanol and basal level of sleepiness. Hum Psychopharmacol 6: 307-311

Rohsenow DJ, Howland J, Arnedt JT, Almeida AB, Greece J, Minsky S, Kempler CS, Sales S (2010) Intoxication with bourbon versus vodka: effects on hangover sleep and next-day neurocognitive performance in young adults. Alcohol Clin Exp Res 34:1-10 
Rohsenow DJ, Howland J, Minsky S, Greece J, Almeida A, Roehrs TA (2007) The acute hangover scale: a new measure of immediate hangover symptoms. Addict Behav 32:1314-1320

Rohsenow DJ, Howland J, Minsky S, Arnedt JT (2006) Effects of heavy drinking by maritime academy cadets on hangover perceived sleep and next-day ship power plant operation. J Stud Alcohol 67:406415

Rosvold HE, Mirsky AF, Sarason I, Bransome ED Jr, Beck LH (1956) A continuous performance test of brain damage. J Consult Psychol 20: 343-350

Rutherford A, Markopoulos G, Bruno D, Brady-Van-den-Bos M (2012) Long-term memory: encoding to retrieval. In: Braisby N, Gellatly A (eds) Cognitive psychology . Oxford University Press, Oxford

Schacter DL (1987) Implicit memory: history and current status. J Exp Psychol 3:501-518

Sjøgren MD, Thomsen AN, Olsen AK (2000) Impaired neuropsychological performance in chronic nonmalignant pain patients receiving long-term oral opioid therapy. J Pain Symptom Manag 19:100-108

Slutske WS, Piasecki TM, Hunt-Carter EE (2003) Development and initial validation of the hangover symptoms scale: prevalence and correlates of hangover in college students. Alcohol Clin Exp Res 27: $1442-1450$

Squire LR (1992) Declarative and nondeclarative memory: multiple brain systems supporting learning and memory. J Cogn Neurosci 4:232-243

Stephens R, Ling J, Heffernan TM, Heather N, Jones K (2008) A review of the literature on the cognitive effects of the alcohol hangover. Alcohol Alcohol 43:163-170

Streufert S, Pogash R, Braig D, Gingrich D, Kantner A, Landis R, Lonardi L, Roache J, Severs W (1995) Alcohol hangover and managerial effectiveness. Alcohol Clin Exp Res 19:1141-1146

Süß HM, Oberauer K, Wittmann WW, Wilhelm O, Schulze R (2002) Working-memory capacity explains reasoning ability-and a little bit more. Intelligence 30:261-288

Swift R, Davidson D (1998) Alcohol hangover: mechanisms and mediators. Alcohol Health Res World 22:54-60
Taylor JL, Dolhert N, Friedman L, Mementhaler M, Yesavage JA (1996) Alcohol elimination and simulator performance of male and female aviators: a preliminary report. Aviat Space Environ Med 67:407413

Törnros J, Laurell H (1991) Acute and hang-over effects of alcohol on simulated driving performance. Blutalkohol 28:24-30

Turgay A, Binder C, Snyder R, Fisman S (2003) Long-term safety and efficacy of risperidone for the treatment of disruptive behavior disorders in children with subaverage IQs. Pediatrics 110:97-109

Vandierendonck A, Liefooghe B, Verbruggen F (2010) Task switching: interplay of reconfiguration and interference control. Psychol Bull 136:601-626

Verbruggen F, Logan GD (2008) Response inhibition in the stop-signal paradigm. Trends Cogn Sci 12:418-424

Verbruggen F, Logan GD (2009) Models of response inhibition in the stop-signal and stop-change paradigms. Neurosci Biobehav Rev 33: 647-661

Verbruggen F, Chambers CD, Logan GD (2013) Fictitious inhibitory differences: how skewness and slowing distort the estimation of stopping latencies. Psychol Sci 24:352-362

Verster JC (2008) The alcohol hangover-a puzzling phenomenon. Alcohol Alcohol 43:124-126

Verster JC, Stephens R, Penning R et al (2010) The alcohol hangover research group consensus statement on best practice in alcohol hangover research. Curr Drug Abuse Rev 3:116-126

Verster JC, van Duin D, Volkerts ER, Schreuder AHCML, Verbaten MN (2003) Alcohol hangover effects on memory functioning and vigilance performance after an evening of binge drinking. Neuropsychopharmacology 28:740-746

Wiese JG, Shlipak MG, Browner WS (2000) The alcohol hangover. Ann Intern Med 132:897-902

Yesavage A, Leirer VO (1986) Hangover effects of aircraft pilots $14 \mathrm{~h}$ after alcohol ingestion: a preliminary report. Am J Psychiatry 143: $1546-1550$ 